University of Nebraska - Lincoln

DigitalCommons@University of Nebraska - Lincoln

7-16-2001

\title{
Evidence for temperature dependent moments ordering in ferromagnetic $\mathrm{NiMnSb}(100)$
}

\author{
C.N. Borca \\ University of Nebraska-Lincoln \\ Takashi Komesu \\ University of Nebraska-Lincoln, tkomesu2@unl.edu \\ Hae-Kyung Jeong \\ University of Nebraska-Lincoln, hjeong@unl.edu \\ Peter A. Dowben \\ University of Nebraska-Lincoln, pdowben@unl.edu \\ Delia Ristoiu \\ CNRS Laboratoire Louis Ne'el, France \\ See next page for additional authors
}

Follow this and additional works at: https://digitalcommons.unl.edu/physicsdowben

Part of the Physics Commons

Borca, C.N.; Komesu, Takashi; Jeong, Hae-Kyung; Dowben, Peter A.; Ristoiu, Delia; Hordequin, Ch.; Nozie`res, J. P.; Pierre, J.; Stadler, Shane; and Idzerda, Y. U., "Evidence for temperature dependent moments ordering in ferromagnetic NiMnSb(100)" (2001). Peter Dowben Publications. 23.

https://digitalcommons.unl.edu/physicsdowben/23

This Article is brought to you for free and open access by the Research Papers in Physics and Astronomy at DigitalCommons@University of Nebraska - Lincoln. It has been accepted for inclusion in Peter Dowben Publications by an authorized administrator of DigitalCommons@University of Nebraska - Lincoln. 


\section{Authors}

C.N. Borca, Takashi Komesu, Hae-Kyung Jeong, Peter A. Dowben, Delia Ristoiu, Ch. Hordequin, J. P. Nozie`res, J. Pierre, Shane Stadler, and Y. U. Idzerda 


\title{
Evidence for temperature dependent moments ordering in ferromagnetic $\mathrm{NiMnSb}(100)$
}

\author{
C. N. Borca, Takashi Komesu, Hae-Kyung Jeong, and P. A. Dowben \\ Department of Physics and Astronomy and the Center for Materials Research and Analysis, Behlen Laboratory of Physics, \\ University of Nebraska —Lincoln, Lincoln, Nebraska 68588-0111 \\ Delia Ristoiu, Ch. Hordequin, J. P. Nozières, and J. Pierre \\ CNRS Laboratoire Louis Néel, 25 avenue des Martyrs, BP 166, 38042 Grenoble CEDEX 09, France
}

\author{
Shane Stadler and Y. U. Idzerda \\ Naval Research Laboratory, Material Physics Branch, Washington, DC 20375
}

(Received 25 April 2001; published 16 July 2001)

\begin{abstract}
From magnetic circular dichroism measurements of the Heusler alloy NiMnSb, in combination with other techniques, we show that a dramatic increase in the Mn and Ni moments occurs below a "crossover" transition temperature of about $80-100 \mathrm{~K}$. This phase transition is well below the Curie temperature of NiMnSb $(\sim 730$ $\mathrm{K})$. While the spin polarization near the Fermi level, from spin-polarized inverse photoemission, remains very high for $k_{\|}=0$, it is unlikely that NiMnSb is a half-metallic ferromagnet near room temperature.

DOI: 10.1103/PhysRevB.64.052409

PACS number(s): 75.25.+z, 71.20.Lp, 73.20.At
\end{abstract}

If both ferromagnetic electrodes are $100 \%$ spin polarized in a tunnel magneto-resistive device, no current will flow in the antiparallel configurations, and the tunnel magnetoresistive (TMR) becomes theoretically infinite. This is one reason why high polarization materials are desirable. Heusler alloys $(\mathrm{NiMnSb}){ }^{1-4}$ colossal magnetoresistance materials $\left[\mathrm{La}_{1-x} \mathrm{Sr}_{x} \mathrm{MnO}_{3},{ }^{5-6} \mathrm{Sr}_{2} \mathrm{FeMoO}_{6}\right.$ (Ref. 7)], and semimetal magnetic oxides $\left[\mathrm{CrO}_{2}\right.$ (Refs. 8-11) and $\mathrm{Fe}_{3} \mathrm{O}_{4}$ (Ref. 12)] are the most cited candidates for $100 \%$ spin polarization. Such systems have been predicted, on the basis of ground state calculations, ${ }^{1-3,5-12}$ to be half-metallic: semiconducting or insulating in spin minority and metallic in spin majority. Compelling evidence of high polarization is available only for $\mathrm{La}_{1-x} \mathrm{Sr}_{x} \mathrm{MnO}_{3}$ (Ref. 13) and $\mathrm{CrO}_{2}$ (Refs. 13 and 14) and then only at temperatures in the region of $4 \mathrm{~K}$ or less.

The Heusler alloys have been particularly important candidates because they exhibit a much higher ferromagnetic Curie temperature $(730 \mathrm{~K})$ than the other potential halfmetallic materials, but experimental evidence of $100 \%$ spin polarization near the Fermi level is sadly absent in NiMnSb. Using Andreev reflection ${ }^{13}$ the spin polarization of $\mathrm{NiMnSb}$ was measured to be $58 \pm 2.3 \%$, in good agreement with a polarization of about $50 \%$ obtained from spin-polarized photoemission. ${ }^{15}$ These polarization values are consistent with a small perpendicular magnetoresistance measured for $\mathrm{NiMnSb}$ in a spin-valve structure, ${ }^{16,17}$ superconducting tunnel junction, ${ }^{18}$ and a tunnel magnetoresistive junction. ${ }^{19}$ Very high polarization values were observed (near 100\% spin asymmetry) near the Fermi level using spin-polarized inverse photoemission of $\mathrm{NiMnSb},{ }^{20}$ but like similar measurements for $\mathrm{La}_{0.67} \mathrm{Sr}_{0.33} \mathrm{MnO}_{3}$ (Ref. 21) and $\mathrm{CrO}_{2}$, ${ }^{22}$ the full Fermi surface was not sampled. ${ }^{20}$

Three explanations have been put forward to explain the smaller than expected polarization observed in the abovementioned experiments. First, the gap in the spin minority band structure is smaller than the expected $0.5 \mathrm{eV}^{4}$ Other possibilities are that there is surface segregation ${ }^{16,18,20,21,23}$ or a variety of defects ${ }^{24}$ making the surface a different material from the bulk. Indeed the segregation or enrichment of the surface has been shown to reduce the polarization of the surface dramatically. ${ }^{20}$ Finally, the half-Heusler alloy $\mathrm{NiMnSb}$ may not be half-metallic ferromagnet above 80 $\mathrm{K},{ }^{25,27}$ though this would not explain the measured low polarization and tunnel magnetoresistance below $80 \mathrm{~K}$. A detailed study of the magnetic and transport properties in bulk single crystals has suggested that, while NiMnSb may be a half-metallic ferromagnet at low temperature, there is a crossover or transition to a normal ferromagnet state around $T=80 \mathrm{~K} .{ }^{27}$ Here, we provide direct evidence that the phase transition at about 80 to $100 \mathrm{~K}$ is associated with a change in the magnetic moment.

Epitaxial (110)MgO/(100)Mo/(100) NiMnSb thin films were grown by facing targets sputtering. ${ }^{28}$ A $1000 \AA$ Sb capping layer was added to prevent oxidation of the $\mathrm{NiMnSb}$ monocrystalline films. Starting with a fully capped layer is important because the surface of $\mathrm{NiMnSb}(100)$ is very fragile. $^{20,23}$ After removal the excess $\mathrm{Sb}$, the stoichiometric surface is prepared ${ }^{20}$ and the LEED pattern as well as x-ray diffraction is consistent with the $5.9 \AA$ (here $6.0 \pm 0.1 \AA$ ) lattice constant of NiMnSb. The surface composition, determined by angle-resolved XPS, was representative of the stoichiometric alloy and the surface was found to be terminated in $\mathrm{MnSb}^{20,23}$

The spin-polarized inverse photoemission (SPIPES) experiments were undertaken as described elsewhere. ${ }^{20}$ The $\mathrm{X}$-ray absorption (XAS) and magnetic circular dichroism (MCD) spectra were recorded by monitoring the sample current, which corresponds to measuring the total electron yield, under a 200 Oe magnetic field pulsed along the in-plane (100) axis. The MCD spectra were recorded by alternating the magnetization direction at each photon energy with a fixed helicity of the incident light. Integrating the MCD signal, we have obtained a quantitative measure of the relative (not absolute) magnetic moment with spin and orbital contributions. In total electron yield mode, the XAS spectra probes approximately 20 to $200 \AA$ in depth, which is an 


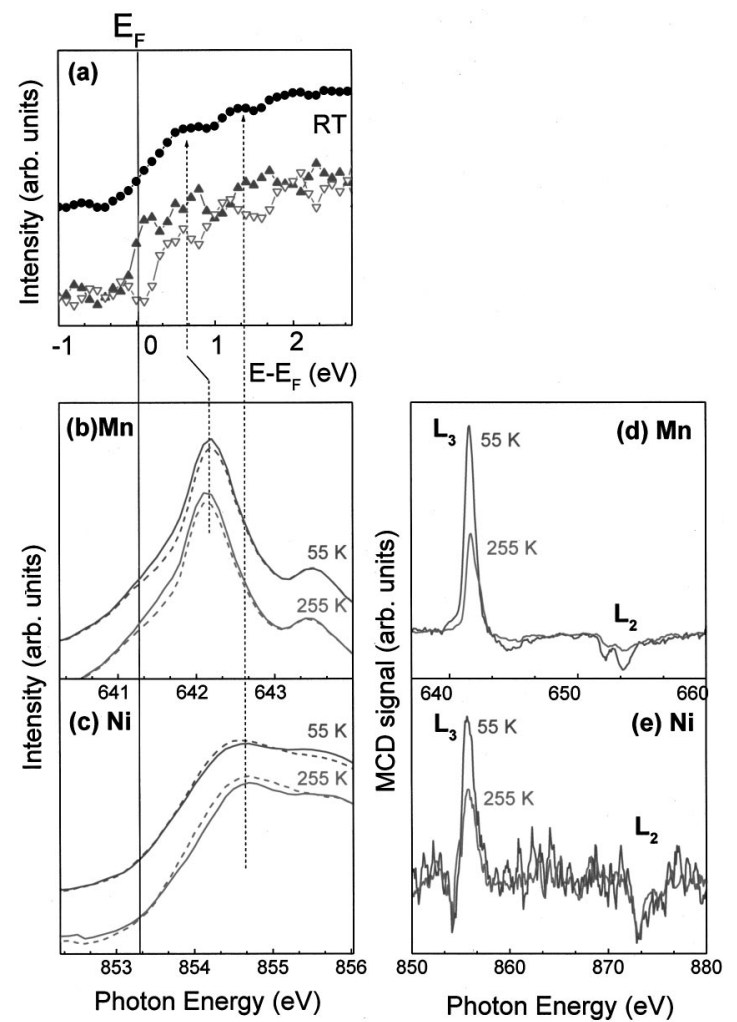

FIG. 1. The comparison of the SPIPES, MCD, and XAS spectra for $\mathrm{NiMnSb}(100)$. The spin-polarized inverse photoemission (SPIPES) ( $\boldsymbol{\Delta}$ spin up, $\nabla$ spin down) and integrated $(\boldsymbol{\bullet})$ data for the stochiometric $\mathrm{NiMnSb}(100)$ clean surface at $300 \mathrm{~K}$ (a) are compared to x-ray absorption results of Mn $2 p$ (b) and Ni $2 p$ (c) electrons. Spectra at two different temperatures are shown for the $\mathrm{x}$-ray adsorption to illuminate the fact that the integrated unoccupied density of states is somewhat insensitive to the phase transition at $80 \mathrm{~K}$. The magnetic circular dichroism signal across both the $L_{2}$ and $L_{3}$ edges of Mn (d) and Ni (e) are shown for two different temperatures to indicate the profound change that occurs between 80 to $100 \mathrm{~K}$ in the spin and orbital moment. The dashed lines indicate the possible corresponding unoccupied states orbitals in SPIPES with the XAS.

intermediate length scale between the bulk and the surface boundary. The polarized neutron scattering results used here for comparison was carried out in the presence of a $4.6 \mathrm{~T}$ applied field as described in Ref. 26.

The spin-polarized unoccupied density of states, as well as the spin-integrated unoccupied density of states (-), of the stoichiometric clean $\mathrm{NiMnSb}(100)$ surface (terminated in $\mathrm{MnSb}$ ) at $293 \mathrm{~K}$, is shown in Fig. 1(a) for the surface Brillouin zone center. The spin-majority states $(\mathbf{\Lambda})$, i.e., parallel to applied magnetic field and spin-minority states $(\nabla)$, i.e., antiparallel to the applied magnetic field, in spin-polarized inverse photoemission, are plotted separately. This spinpolarized inverse spectrum of the unoccupied density of states can be compared to the x-ray absorption spectra (XAS) for Mn $2 p$ [Fig. 1(b)] and for Ni $2 p$ [Fig. 1(c)], due to the fact that both techniques (ignoring the core hole in the case of XAS) generally probe the same unoccupied states. Tuning the photon energy to the $2 p$ core level threshold, we expect core to bound state excitations to the unoccupied hybridized

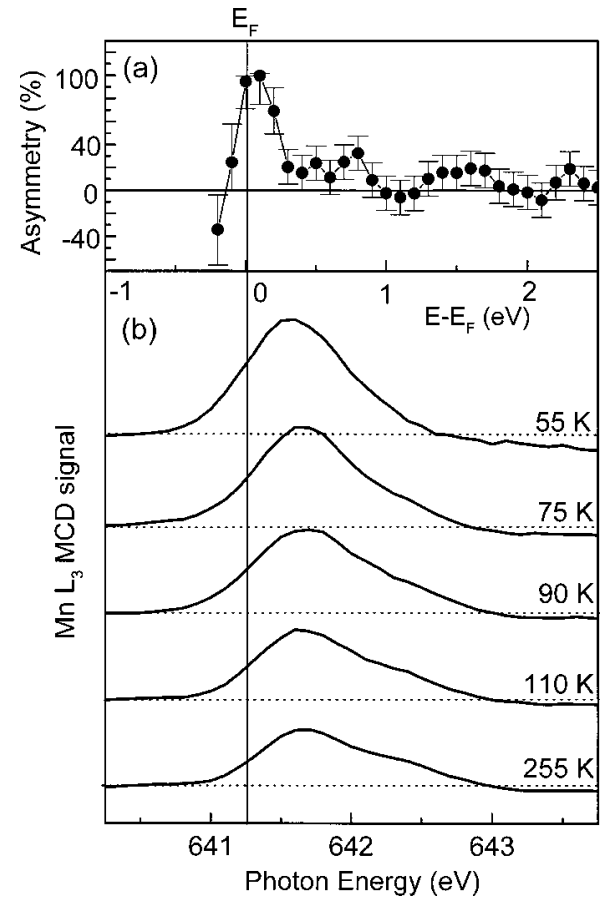

FIG. 2. The polarization asymmetry from SPIPES (at the elevated temperature of $300 \mathrm{~K}$ ) (a) is compared to the temperature dependent magnetic circular dichroism signal data across Mn $2 p$ (b) core levels. The MCD signal is shown for several different temperatures, increasing as the temperature decreases.

$3 d$ levels closest to the Fermi level.

For both MCD and XAS spectra, the conduction band edge (the approximate Fermi energy) for the XAS spectra is assigned on the basis of the experimentally measured core level photoemission binding energies for $\operatorname{NiMnSb}(100)$ : $640.2 \mathrm{eV}$ for the Mn $2 p_{3 / 2}$ and $853.3 \mathrm{eV}$ for the Ni $2 p_{3 / 2}$ (Fig. 1). In the XAS spectra, the unoccupied states are shifted to slightly higher energies above $E_{F}$ than in SPIPES, as expected from the perturbation due to the Coulombic interaction of the photoexcited electron with the core hole. Nonetheless, from this comparison, we can make a rough assignment of the unoccupied states in inverse photoemission. The density of states just above $E_{F}$ for $\mathrm{NiMnSb}(100)$ is largely $\mathrm{Mn}$ in origin (Fig. 1). While generally consistent with other XAS/MCD studies, ${ }^{3,29}$ our Mn XAS spectra are characteristic of $\mathrm{Mn} d^{5}$ (and could include the expected $d^{4}$ contributions) $)^{29}$ at all temperatures from 45 to $250 \mathrm{~K}$.

The polarization of the $\mathrm{NiMnSb}(100)$ surface is determined by the difference of spin up and down in SPIPES [Fig. 2(a)]. Subject to the necessary selection rules, core excitation spectra reflect the joint density of states between the core level and the unoccupied states. These results are compared with the MCD [the difference between circular light XAS signals taken with opposite magnetization directions, indicated Figs. 1(d) and 1(e)] across the Mn $2 p_{3 / 2}$ core in Fig. 2(b). The large polarization asymmetry, and the strong contribution from the Mn spin and orbital moments at remanence, are apparent in the region close to the Fermi level (nearly $100 \%$ above background for $\mathbf{k}_{\|}=0$ and $T=300 \mathrm{~K}$ ), as seen in Fig. 2. The remanent band moment is localized on 


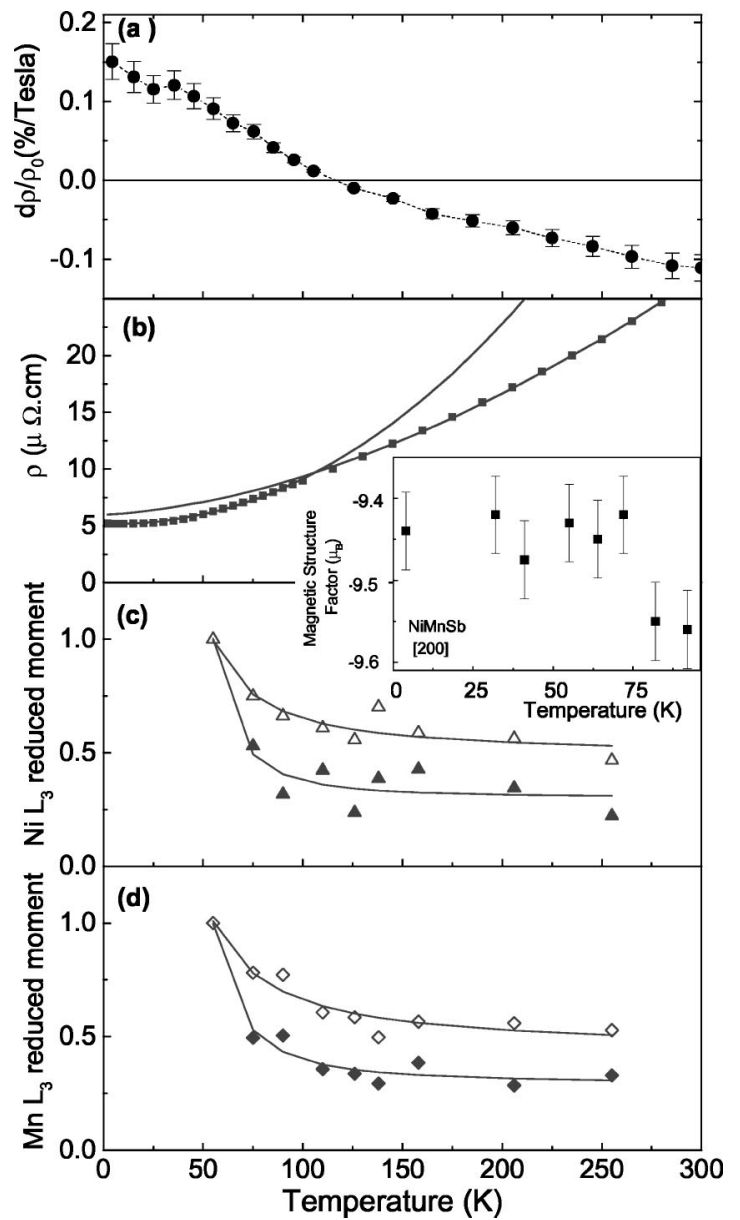

FIG. 3. The longitudinal magnetoresistance slope $\left(d \rho / \rho_{0}\right)$ taken between 2 and $4 \mathrm{~T}$ (a), compared to the resistance (b), the relative $\mathrm{Ni}$ moment derived from MCD (c), and relative Mn moment (d) derived from MCD. The relative moment contains both spin and orbital contributions from the $L_{3}$ edge (open symbols) and sum of both the $L_{3}$ and $L_{2}$ edges (closed symbols) and the data are fit to an Arrenhius expression. Note that the changes in the moment observed in MCD compare well with the onset of changes in the magnetoresistance and resistance in $\mathrm{NiMnSb}$, as well as the change in the magnetic structure factor for the $\langle 200\rangle$ peak in neutron scattering shown as an inset, as adapted from Ref. 26. The $T^{2}$ and $T^{1.65}$ curves fitted to the resistance data are as described in the text.

the $\mathrm{Mn}$ and $\mathrm{Ni}$ sites, as the moment on $\mathrm{Sb}$ should be of the order of $0.1 \mu_{B}$ from band calculations.

While polarization at the Brillouin zone center $\left(\mathbf{k}_{\|}=0\right)$ does correspond to long-range magnetic order, it is not clear that the polarization is, in fact, easily related to the magnetic moment for any given system. As the temperature is decreased below $80 \mathrm{~K}$, the MCD exhibits a huge increase in the relative spin and orbital moment, as seen in Fig. 2 and summarized in Fig. 3. Yet in XAS, the apparent density of states near the Fermi level (threshold) does not change significantly for $\mathrm{Mn}$ or $\mathrm{Ni}$. Therefore, the polarization of $\mathrm{NiMnSb}(100)$ close to $100 \%$ at an elevated finite temperature (300 K here), at the Brillouin zone center $\left(\mathbf{k}_{\|}=0\right)$, is not a good indicator of the maximum possible remanent band moment or indeed the total "integrated" band polarization in the NiMnSb system. The results, presented here, are clear proof that any angle-resolved spin-polarized photoemission or spinpolarized inverse photoemission measurements, with limited wave vector sampling, cannot be taken as sufficient evidence of half-metallic ferromagnetic character if the sample is crystalline or is polycrystalline with texture growth.

Because magnetic circular dichroism is sensitive to band structure polarization (dependent upon symmetry and wave vector), the remanent unoccupied band polarization, the magnetocrystalline anisotropy (the crystal field), ${ }^{30,31}$ the excitation cross sections (particularly in nontransmission absorption geometries) and an often undetermined surface contribution, MCD does not accurately probe the saturation local moment of the bulk material. A more accurate measure of the local bulk sample moment can be obtained from neutron scattering (though this is a less sensitive measure of the global moment that includes local fluctuations). The Fourier transform of the magnetic scattering factor, from neutron scattering, again demonstrates that most of the moment is localized on the Mn sites. ${ }^{26}$ The total moment from high field magnetometry (at $10 \mathrm{~K}$ ) is $4.025 \mu_{B}$, consistent with halfmetallic character (we attribute the possible excess moment to altered stoichiometry and/or polarization of the inner core shells). From polarized neutron scattering we find the local moment on the $\mathrm{Mn}$ at $15 \mathrm{~K}, 3.79 \pm 0.02 \mu_{B}$, falls to 3.55 $\pm 0.02 \mu_{B}$ at $260 \mathrm{~K}$, while the local moment on the Ni sites changes little, with values varying from $0.18 \pm 0.02 \mu_{B}$ at 15 $\mathrm{K}$ to $0.19 \pm 0.01 \mu_{B}$ at $260 \mathrm{~K}$. The fact that the local moments change, even under high field conditions, suggests that there is a change in the moment coupling between $\mathrm{Mn}$ and $\mathrm{Ni}$ at about $80 \mathrm{~K}$. At higher temperatures above 80 to $100 \mathrm{~K}$, the saturation moment is lower than that expected for a halfmetallic ferromagnet.

The observed increase in the local moment, on the $\mathrm{Mn}$ in neutron scattering, and band moment on the $\mathrm{Mn}$ and $\mathrm{Ni}$ atoms in NiMnSb below 80 to $100 \mathrm{~K}$ in MCD [Figs. 3(c) and $3(\mathrm{~d})]$ are consistent with the increase in the $\left(d \rho / \rho_{0}\right)$ longitudinal magnetoresistance below $80 \mathrm{~K}$ [shown in Fig. 3(a)], and the increase in the $\langle 200\rangle$ neutron magnetic scattering factor $^{26}$ (indicated as in the inset of Fig. 3). The negative value of the longitudinal magnetoresistance indicates that spin disorder contributions dominate above $100 \mathrm{~K}$, when a spin-flip scattering channel is opened. The spin-flip scattering channel cannot be reconciled with half-metallic character. The resistance of NiMnSb with temperature fits a $T^{1.65}$ power law above $80 \mathrm{~K}$ (close to $T^{3 / 2}$ ) but follows a $T^{2}$ power law below $80 \mathrm{~K}$, as shown in Fig. 3(b) is also consistent with a crossover in magnetic ordering at 80 to $100 \mathrm{~K}$, though the material remains ferromagnetic. Fitting the MCD $L_{3}$ edge data in Fig. 3 to an Arrhenius expression (consistent with moment fluctuations), indicates that the critical temperature for the classical ferromagnetic to half-metallic ferromagnetic (moment ordering) phase transition is between 80 to $100 \mathrm{~K}$.

The changes in the magnetic structure factor(s) suggest a loss in the strong local moment ordering and/or alignment of the $\mathrm{Ni}$ atoms. The structure factors suggest a subtle rearrangement of the magnetization density between $\mathrm{Ni}$ and $\mathrm{Mn}$. The transfer of only a small amount of electron density, say just a few hundredth of an electron to the down-spin subband or from $\mathrm{Mn}$ to $\mathrm{Ni}$, may not result in much change in 
either the total moment or the Mn saturation moment (say by about $0.05 \mu_{B}$ ), but could substantially change the net band polarization near the Fermi level. This effect tends to appear as though there is a failure to completely saturate the magnetization in the MCD measurements, taken at remanence, with increasing temperature. The large change in the MCD signal derived moment compared to the smaller change in neutron scattering moment suggests a large change in the magnetocrystalline anisotropy and band polarization with temperature, though the extremely low effective Debye temperature in the surface region ${ }^{32}$ presents a complication to the interpretation of the MCD data that cannot be ignored. Nearest-neighbor moment fluctuations would reconcile the spin-polarized inverse photoemission and the MCD with the neutron scattering as in the spin-polarized inverse photoemission nearest-neighbor interactions would only be observed away from the Brillouin zone center.

While it has been suggested that NiMnSb is a halfmetallic system, ${ }^{1-3}$ it is certainly clear, from the data presented here, that $\mathrm{NiMnSb}$ is not a half-metallic ferromagnet above the transition at about $80-100 \mathrm{~K}$, though still at tem- peratures well below the ferromagnetic Curie temperature of $\sim 730 \mathrm{~K}$. Amongst the other potential half-metallic systems, the manganese perovskites have exhibited a number of phase transitions well below the ferromagnetic $T_{c}{ }^{32,33}$ The presence of these phase transitions (affecting the moment in the case of $\mathrm{NiMnSb}$ ) makes clear that finite temperature calculations and measurements that probe the polarization of Fermi level crossings are essential to establish half-metallic ferromagnetism at any appreciable temperature, particularly for the surface. Neither $100 \%$ polarization at one $\mathbf{k}$ point nor $T^{3 / 2}$ temperature dependent resistance (sometimes taken as indicative of a half-metallic ferromagnet ${ }^{2}$ ) are good indicators of half-metallic character above $80 \mathrm{~K}$.

This work was supported by NSF through Grant No. DMR-98-02126, the Center for Materials Research and Analysis (CMRA) and the Nebraska Research Initiative at the University of Nebraska, and the Region Rhone-Alpes through the "Nanotechnologie" program under Contract No. PR97024.
${ }^{1}$ R. A. de Groot, F. M. Mueller, P. G. van Engen, and K. H. J. Buschow, Phys. Rev. Lett. 50, 2024 (1983).

${ }^{2}$ V. Yu. Irkhin and M. I. Katsnel'son, Phys. Usp. 37, 659 (1994).

${ }^{3}$ I. Galanakis, S. Ostanin, M. Alouani, H. Dreysse, and J. M. Wills, Phys. Rev. B 61, 4093 (2000).

${ }^{4}$ J.-S. Kang, J.-G. Park, C. G. Olson, S. J. Youn, and B. I. Min, J. Phys.: Condens. Matter 7, 3789 (1995).

${ }^{5}$ W. E. Pickett and D. J. Singh, Phys. Rev. B 53, 1146 (1996).

${ }^{6}$ D. J. Singh and W. E. Pickett, Phys. Rev. B 57, 88 (1998).

${ }^{7}$ K.-I. Kobayashi et al., Nature (London) 395, 677 (1998).

${ }^{8}$ K. Schwarz, J. Phys. F: Met. Phys. 16, L211 (1986).

${ }^{9}$ H. van Lueken and R. A. de Groot, Phys. Rev. B 51, 7176 (1995).

${ }^{10}$ M. A. Korotin, V. I. Anisimov, D. I. Khomskii, and G. A. Sawatzky, Phys. Rev. Lett. 80, 4305 (1998).

${ }^{11}$ S. P. Lewis, P. B. Allen, and T. Sasaki, Phys. Rev. B 55, 10253 (1997).

${ }^{12}$ R. A. de Groot and K. H. J. Bischow, J. Magn. Magn. Mater. 54-57, 1377 (1986).

${ }^{13}$ R. J. Soulen et al., Science 282, 85 (1998).

${ }^{14}$ R. Weisendanger, H.-J. Güntherodt, G. Güntherodt, R. J. Gambino, and R. Ruf, Phys. Rev. Lett. 65, 247 (1990).

${ }^{15}$ G. L. Bona, F. Meier, M. Taborelli, E. Bucher, and P. H. Schmidt, Solid State Commun. 56, 391 (1985).

${ }^{16}$ J. A. Caballero, A. C. Reilly, Y. Hao, J. Bass, W. P. Pratt, F. Petroff, and J. R. Childress, J. Magn. Magn. Mater. 198-199, 55 (1999).

${ }^{17}$ R. Kabani, M. Terada, A. Roshko, and J. S. Moodera, J. Appl. Phys. 67, 4898 (1990).

${ }^{18}$ C. T. Tanaka, J. Nowak, and J. S. Moodera, J. Appl. Phys. 86,
6239 (1999).

${ }^{19}$ C. Tanaka, J. Nowak, and J. S. Moodera, J. Appl. Phys. 81, 5515 (1997).

${ }^{20}$ D. Ristoiu, J. P. Nozières, C. N. Borca, T. Komesu, Hae-kyung Jeong, and P. A. Dowben, Europhys. Lett. 49, 624 (2000).

${ }^{21}$ J.-H. Park, E. Vescovo, H.-J. Kim, C. Kwon, R. Ramesh, and T. Venkatesan, Nature (London) 392, 794 (1998).

${ }^{22}$ K. P. Kämper, W. Schmitt, G. Güntherodt, R. J. Gambino, and R. Ruf, Phys. Rev. Lett. 59, 2788 (1987).

${ }^{23}$ Delia Ristoiu, J. P. Nozières, C. N. Borca, B. Borca, and P. A. Dowben, Appl. Phys. Lett. 76, 2349 (2000).

${ }^{24}$ D. Orgassa, H. Fujiwara, T. C. Schulthess, and W. H. Butler, Phys. Rev. B 60, 13237 (1999).

${ }^{25}$ C. Hordequin, J. Pierre, and R. Currat, J. Magn. Magn. Mater. 162, 75 (1996).

${ }^{26} \mathrm{Ch}$. Hordequin, E. Lelievre-Berna, and J. Pierre, Physica B 234236, 602 (1997)

${ }^{27}$ Ch. Hordequin, D. Ristoiu, L. Ranno, and J. Pierre, Eur. Phys. J. B 16, 287 (2000).

${ }^{28}$ D. Ristoiu, J. P. Nozières, and L. Ranno, J. Magn. Magn. Mater. 219, 97 (2000).

${ }^{29}$ B. T. Thole, R. D. Cowan, G. A. Sawatzky, J. Fink, and J. C. Fuggle, Phys. Rev. B 31, 6856 (1985).

${ }^{30}$ J. Stöhr and H. König, Phys. Rev. Lett. 75, 3748 (1995).

${ }^{31}$ H. Dürr et al., Phys. Rev. Lett. 76, 3464 (1996).

${ }^{32}$ C. N. Borca, D. Ristoiu, Takashi Komesu, Hae-kyung Jeong, Ch. Hordequin, J. Pierre, J. P. Nozieres, and P. A. Dowben, Appl. Phys. Lett. 77, 88 (2000).

${ }^{33}$ M. Imada, A. Fujimori, and Y. Tokura, Rev. Mod. Phys. 70, 1039 (1998). 